\title{
Soy diet worsens heart disease in mice
}

\author{
Brian L. Stauffer,1,2 John P. Konhilas, ${ }^{2}$ Elizabeth D. Luczak, ${ }^{2}$ and Leslie A. Leinwand ${ }^{1,2}$ \\ ${ }^{1}$ Department of Medicine, Division of Cardiology, University of Colorado Health Sciences Center, Denver, Colorado, USA. ${ }^{2}$ Department of Molecular, \\ Cellular, and Developmental Biology, University of Colorado at Boulder, Boulder, Colorado, USA.
}

\begin{abstract}
We report that dietary modification from a soy-based diet to a casein-based diet radically improves disease indicators and cardiac function in a transgenic mouse model of hypertrophic cardiomyopathy. On a soy diet, males with a mutation in the $\alpha$-myosin heavy chain gene progress to dilation and heart failure. However, males fed a casein diet no longer deteriorate to severe, dilated cardiomyopathy. Remarkably, their LV size and contractile function are preserved. Further, this diet prevents a number of pathologic indicators in males, including fibrosis, induction of $\beta$-myosin heavy chain, inactivation of glycogen synthase kinase $3 \beta$ (GSK3 $\beta$ ), and caspase- 3 activation.
\end{abstract}

\section{Introduction}

The promise of nutritional genomics in considering the pathogenesis and treatment of disease is beginning to be recognized (1-5). For example, soy-rich diets are perceived to be generally beneficial from a health standpoint, particularly in the context of the cardiovascular system (6). This is exemplified by the fact that American consumers will spend $\$ 4.7$ billion on soy foods and dietary supplements. However, there has not been a systematic experimental evaluation of how individuals with defined genetics respond to different diets and how sex might modify this interaction. One starting point to evaluate the effect of a particular diet such as soy is the laboratory rodent, where the standard diet is soy-based. Potent physiologic effects of soy diets have been demonstrated in both laboratory and clinical settings. Some of these effects have been reported to be beneficial and some may have adverse consequences. Among the beneficial properties are the prevention of cancer and a lowering of cholesterol (7). Among the potentially adverse effects are an increase in androgen levels and a decrease in thyroid peroxidase $(8,9)$. Many of the physiologic effects of a soy diet have been attributed to the soy isoflavones or phytoestrogens, and there are many experimental studies that have studied the effects of genistein and daidzein, the 2 most prominent isoflavones in soy (10). Indeed, soy isoflavones improve hyperlipidemia and cardiovascular disease associated with abnormalities in lipid metabolism via activation of $\operatorname{PPAR} \alpha(11,12)$. In human and rodent heart failure, PPAR $\alpha$ activity is attenuated $(13,14)$. However, a recent large epidemiologic study revealed no beneficial effect of dietary phytoestrogens on the incidence of clinical coronary or cerebrovascular events in women (15). Despite this focus on phytoestrogens, soy has many complex nutrients and is consumed as a major part of the diet in many cultures. Further, decades of literature on experimental laboratory rodents have been in the context of a soy-based diet (rather than a diet supplemented by dietary phytoestrogens), and thus it is of great interest to examine the interaction of the soy diet and genetic models of disease. In the current study, we have asked what the impact of diet is on a genetic mouse model of cardiac hypertrophy and how sex might modify such a proposed interaction.

Nonstandard abbreviations used: BW, body weight; ER, estrogen receptor; GSK3 $\beta$, glycogen synthase kinase $3 \beta$; HCM, hypertrophic cardiomyopathy; HW, heart weight; $\mathrm{MyHC}$, myosin heavy chain; TL, tibia length; VW, ventricular weight.

Conflict of interest: The authors have declared that no conflict of interest exists.

Citation for this article: J. Clin. Invest. 116:209-216 (2006).

doi:10.1172/JCI24676.
Myocardial hypertrophy in response to a disease stimulus consists initially of compensatory myocellular enlargement. However, the heart ultimately reaches a point where the stress overwhelms compensatory processes, and ventricular chamber enlargement, wall thinning, and impaired contractile function ensue, leading to heart failure. The myocardial mechanisms underlying this transition are thus far unknown.

Sex-specific variation in myocardial hypertrophy and progression to heart failure have been clearly documented over the past several decades $(16,17)$. In response to a disease stimulus (hypertension, ref. 18; valvular disease, ref. 17; sarcomeric mutations, ref. 19 ; and aging, refs. 20,21), both sexes initially develop LV hypertrophy. However, men subsequently develop heart failure with chamber dilation, wall thinning, and impaired contractile function. Women develop heart failure with preserved LV contractile function. The sex difference in cardiac function favors the survival of women with heart failure $(22,23)$.

While these sex-differences in clinical cardiac disease are reasonably well characterized, there is a dearth of mechanistic information about the triggers of increased mortality. Several studies have evaluated the effects of sex hormones $(24,25)$ and phytoestrogens (15) on the cardiovascular system in humans, predominantly investigating effects on heart attack or stroke, disorders more associated with abnormalities of blood vessels. While estrogen and testosterone receptors have been identified in the myocardium $(21,26,27)$, less is known about the influence of sex hormones on the myocardium.

We have developed a genetic mouse model of hypertrophic cardiac disease that exhibits the sex-dependent phenotypic characteristics documented in clinical populations (28). That is, while females preserve cardiac contractile function and continue to increase their cardiac mass, male mice develop thin ventricular walls and have poorly contractile hearts. This hypertrophic cardiomyopathic (HCM) mouse expresses a mutant myosin heavy chain (MyHC) transgene in the heart, and all of the characterization has been performed on animals fed a soy diet (29). We hypothesized that phytoestrogens influence cardiac growth and that the standard rodent diet plays an influential role in the development of this dilated, poorly contractile phenotype in male mice. We tested this hypothesis by comparing HCM mice and WT littermate controls of both sexes consuming a standard soy-based diet, a caseinbased diet $(30,31)$, or a casein diet supplemented with daidzein and genistein. Further, we determined the effect of diet on several aspects of known hypertrophic signaling. 
A

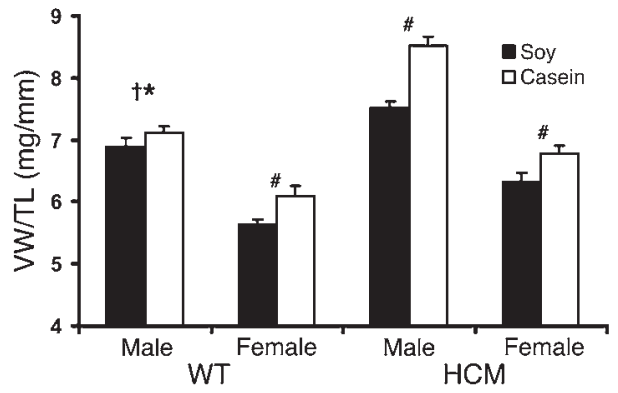

B

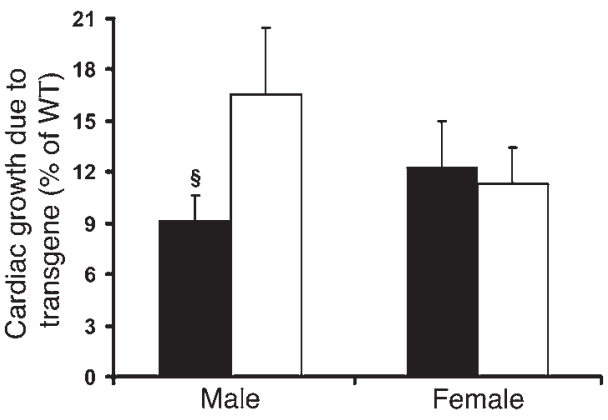

\section{Results}

Diet and cardiac growth. Diet was found to significantly affect cardiac mass. Ventricular weight (VW) (RV and $\mathrm{LV})$ was normalized to tibia length (TL) (Figure 1A). As expected, the HCM animals had higher VW/TL than their sex-matched WT controls fed the same $\operatorname{diet}(P<0.005$ for all 4 groups). Also, as expected, male animals had larger hearts than female animals $(P<0.0001$ for all 4 groups). However, HCM animals had significantly more hypertrophy on the casein diet than on the soy diet $(P<0.05$ each). The male HCM animals fed a casein-based diet had greater cardiac hypertrophy (17\%) than the HCM males fed a soy diet (9\%) when compared with control mice. In contrast, diet did not have an effect on hypertrophy in female HCM animals (Figure 1B). As might be expected, diet also influenced body weight (BW). All mice consuming the casein diet were significantly heavier than those on the soy diet. Body composition, evaluated by dual-energy $\mathrm{x}$-ray absorptiometry (DEXA), demonstrated no significant diet-dependent differences in lean BW; however, the animals consuming the casein diet had higher body fat than those consuming the soy-based diet. Importantly, normalization of VW for BW did not influence the differences between the transgenic groups noted above.

Dietary estrogens and cardiac growth. There are several components that are different between the soy and casein diets. We investigated the possibility that phytoestrogens contribute to differences in cardiac growth between the diets. The phytoestrogens daidzein and genistein were added to the casein diet. This supplemented diet was fed to male and female WT and HCM mice and compared with mice consuming the standard soy and casein diets. There was no significant effect of diet on heart weight/BW (HW/BW) ratios in either the male or female WT mice at 2 months of age. However, there was a significant effect of diet on $\mathrm{HW} / \mathrm{BW}$ ratio in the male and female HCM animals at this early time point $(P<0.01)$. Moreover, the influence of diet was observed in a sex-dependent fashion (sex $\times$ diet interaction, $P<0.05$ ). The influence of adding phytoestrogen to the casein diet was greater in the male mice, augmenting the $\mathrm{HW} / \mathrm{BW}$ ratio by approximately $6 \%$, while this addition

\section{Figure 1}

Cardiac growth due to transgene expression is modified by sex and diet at 8 months of age. (A) VW normalized to body growth, assessed by TL. * Significant transgene effect with each sex and diet combination $\left(P<0.005\right.$ for each). ${ }^{+}$Significant sex effect by diet in both WT and HCM mice $(P<0.0001)$. "Significant diet effect $(P<0.05)$ between groups except in WT males. (B) There is a diet-dependent difference in the degree of hypertrophy in the male mice while there is no diet-dependent difference in the female animals. $\$ P<0.01$ versus male casein. $n=21-35$ in each group. HCM, transgenic HCM mice. Mean \pm SEM.

attenuated cardiac growth in the female HCM mice by $6 \%$ (Figure 2). Importantly, cardiac growth was lower in the HCM animals of both sexes consuming the standard soy diet, and the addition of the phytoestrogens to the casein diet did not completely recapitulate this phenotype.

Endogenous sex steroids and cardiac growth. The influence of the dietary compounds may occur through estrogenic effects on endogenous sex steroid receptors. These receptors have been implicated in cardiac growth in other models $(32,33)$. We investigated the possibility that diet contributes to the phenotype via a sex steroid receptor-mediated mechanism. Male and female HCM mice on the casein diet underwent prepubertal surgical gonadectomy with subsequent placebo, estrogen, or testosterone supplementation or sham operation. Gonadectomy with placebo supplementation (absence of all sex steroids) resulted in lower cardiac mass regardless of sex compared with sham-operated animals (males: $3.37 \pm 0.19 \mathrm{mg} / \mathrm{g} \mathrm{BW}$ versus $4.16 \pm 0.37 \mathrm{mg} / \mathrm{g}$; females: $2.93 \pm 0.21 \mathrm{mg} / \mathrm{g}$ versus $3.45 \pm 0.90 \mathrm{mg} / \mathrm{g}$ ). Testosterone supplementation of the females $(4.47 \pm 0.10 \mathrm{mg} / \mathrm{g})$ and estrogen supplementation of the males $(3.93 \pm 0.27 \mathrm{mg} / \mathrm{g})$ caused cardiac mass to be similar to the opposite sex sham-operated animals. For comparative numbers, see those noted above. In summary, lack of sex hormones results in lower $\mathrm{HW} / \mathrm{BW}$ ratios in both sexes, and testosterone and estrogen appear to be primary determinants of normalized heart mass.

Diet and in vivo cardiac function. Cardiac contractile function evaluated by echocardiography was profoundly affected by diet in male HCM animals (Figure 3). At 8 months of age, HCM males fed a soy diet had significantly depressed contractile function $(P<0.0001)$ while animals fed a casein diet were indistinguishable from WT.

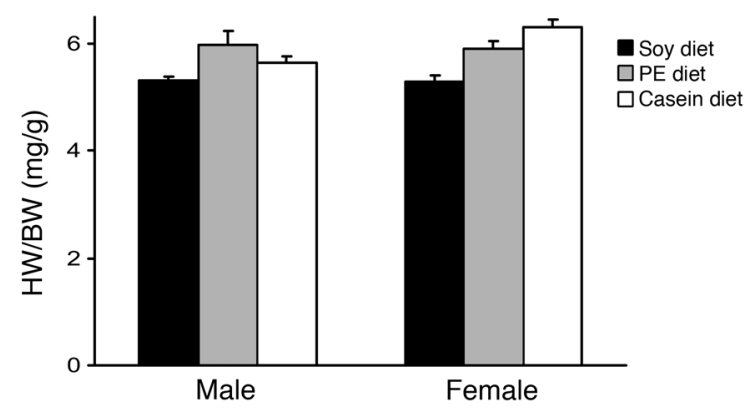

Figure 2

Cardiac growth due to diet in male and female HCM mice consuming a standard soy diet, a casein, phytoestrogen-free diet, or a casein diet supplemented with the phytoestrogens daidzein and genistein (PE diet) was found to occur in a sex-dependent fashion. ANOVA main effects: diet, $P<0.0001$; sex $\times$ diet, $P<0.05 . n=12-19$ in each group except in those on the phytoestrogen-supplemented diet $(n=6)$. 

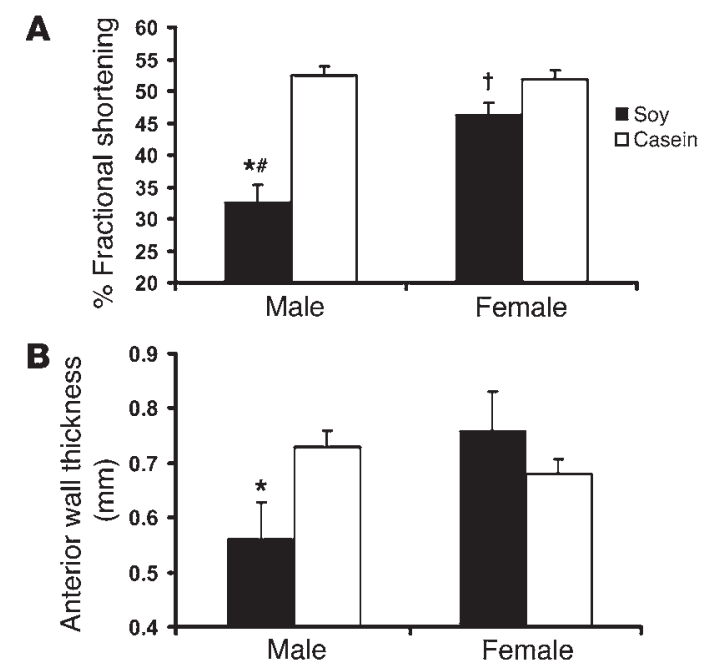

The decrease in contractile function (Figure 3A) was accompanied by significant wall thinning (Figure $3 \mathrm{~B}$ ) and $\mathrm{LV}$ chamber dilation. Thus, a soy-based diet has a very significant detrimental effect on cardiac function in this model of hypertrophic disease.

Diet and blood pressure in vivo. Estrogen is associated with improvements in endothelium-mediated arterial vasodilation, a marker of vascular health. Oral genistein supplementation has also been shown to improve endothelial function in humans (5). Alterations in endothelial and arterial function may lead to alterations in blood pressure that influence the development of cardiac hypertrophy. We therefore sought to determine whether the sex- and diet-dependent differences were due to differences in systemic blood pressure. In vivo hemodynamics with Millar catheterization revealed no significant differences in mean, systolic, or diastolic arterial pressure (data not shown). The lack of influence of sex or diet on blood pressure suggests a primary myocardial influence of diet on the differences observed in cardiac function.

Diet and myocardial fibrosis and myocellular disarray. The effect of diet on histopathology as evidenced by collagen deposition and myofilament disorganization was evaluated at 8 months of age using picrosirius red staining. Fibrosis and myocellular disarray can be visualized under polarization light microscopy by yellow/ orange birefringence and lack of green sarcomeric birefringence, respectively. Highly ordered sarcomeres have light-green birefringent properties (Figure 3). Increased collagen deposition causes increased myocardial stiffness and is associated with myocellular disarray. Both of these features have been implicated in myocardial contractile dysfunction (34-36). HCM animals display an increase in collagen deposition and myocellular disarray compared with WT (Figure 4). This was evidenced by a transition from predominantly thin collagen fibrils (green birefringence) in the WT controls to predominantly thick collagen fibers (yellow/orange birefringence) in the HCM male and female mice (Figure 4 and data not shown). The lack of uniform myofilament birefringence in the HCM sections is also indicative of an increase in myocellular disarray. Most importantly, there was a distinctly worse histopathologic phenotype in the soy HCM males when compared with the casein HCM males. We observed no significant difference in fibrosis and disarray among diets in the female HCM mice. Moreover, the histopathologic phenotype was indistinguishable between the sexes on the casein diet. The increased fibrosis and myocellular disarray

\section{Figure 3}

In vivo cardiac function assessed by echocardiography in HCM mice at 8 months of age. (A) Decreased contractile function (\% fractional shortening) is observed in male HCM mice consuming the soy diet. The impairment is ameliorated on the casein diet. (B) The functional impairment is associated with thinning of the anterior wall of the LV. These abnormalities are prevented by changing the animals to a phytoestrogen-free diet. $n=5-13$ in each group. ${ }^{*} P<0.0001$ versus male casein; ${ }^{\dagger} P<0.05$ versus female casein; ${ }^{\prime} P<0.05$ versus same diet, alternate sex. Mean \pm SEM.

observed in the male HCM mice consuming the soy-based laboratory diet seem likely to contribute to the contractile dysfunction in this experimental group.

Diet and $\beta-M y H C$ protein synthesis. Heart failure in rodents and humans is associated with increased levels of the slower, more energy efficient myosin motor protein, $\beta$-MyHC. $\alpha$-MyHC, the predominant isoform in murine hearts, declines with worsening heart failure and is accompanied by increases in $\beta$-MyHC (37). An increase in $\beta$-MyHC protein content acts in a dominant fashion and is one of the mechanisms underlying myocardial contractile dysfunction $(38,39)$. Additionally, $\beta$-MyHC content can be used as an objective assessment of disease severity. We measured the $\beta$-MyHC content of the LV at 8 months of age (Figure 5, A and B). Because contractile function was abnormal in the male HCM mice consuming the soy diet, we hypothesized that more $\beta$-MyHC protein would be observed in this group. Indeed, male HCM mice fed the soy diet expressed more $\beta$-MyHC than the female HCM mice, consistent with the more severe disease state in males. In addition, there was strikingly lower $\beta$-MyHC content in the male HCM mice consuming the casein diet. This is of particular interest since the absolute and normalized heart weights were greater in the casein-fed mice. This suggests that the growth that occurred in the HCM hearts due to the casein diet is a beneficial (or physiologic) hypertrophy. The increased $\beta$-MyHC protein expression may be a mechanism contributing to the impairment in systolic function in the male HCM mice on the soy diet.

Diet and the IGF-1/Akt/glycogen synthase kinase 3 protein kinase cascade. Since IGF-1 treatment of cardiac myocytes in culture increases $\beta$-MyHC synthesis (40, 41), we explored the IGF-1/Akt/glycogen synthase kinase 3 (IGF-1/Akt/GSK3) cascade. Sex-dependent differences
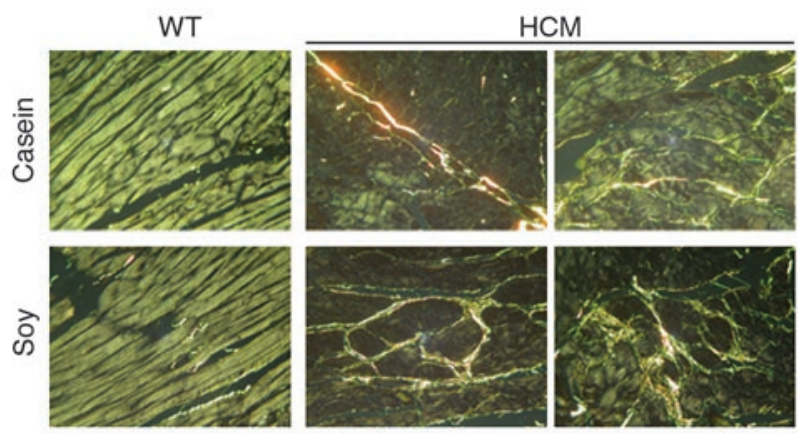

\section{Figure 4}

Cardiac fibrosis is a mechanism behind contractile dysfunction in heart failure. An improvement in cardiac fibrosis is observed in the male HCM mice consuming a casein diet. $n=5$ in each group. Increased collagen deposition in the male HCM mice on the soy diet may contribute to the contractile dysfunction observed by echocardiogram. 


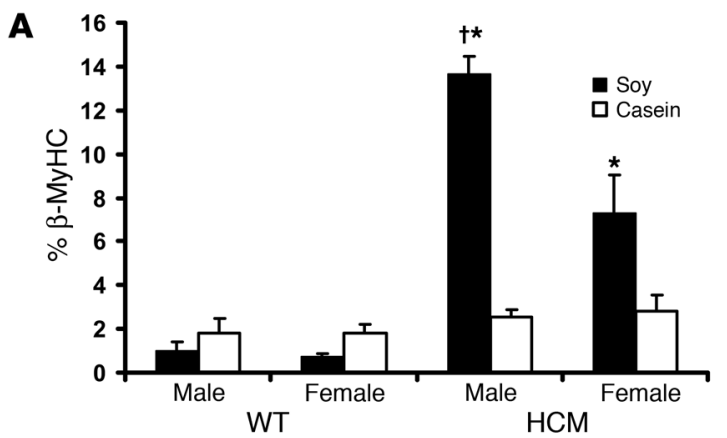

B
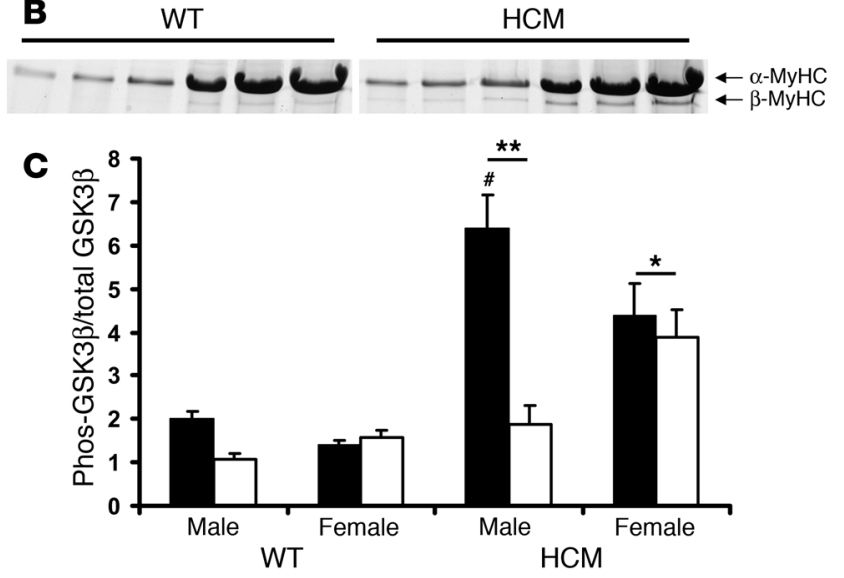

in the IGF-1/Akt/GSK3 $\beta$ pathway have been observed in humans and in animal models of cardiac disease (42). Several animal models have indicated that this pathway is involved in cardiac hypertrophy via phosphorylation of Akt (protein kinase B; activation) and GSK3 $\beta$ (inactivation) $(43,44)$. Increased nuclear localization of phospho-Akt has been observed in the myocardium of premenopausal women as well as in response to estrogen stimulation in cultured cardiomyocytes (42). IGF-1 has been identified as one of several potential ligands responsible for estrogen-independent activation of estrogen receptors (ERs) $(45,46)$. Based on this information, we evaluated GSK3 $\beta$, a terminal protein in this cascade, for changes in activity as a potential protein kinase leading to the sex and diet differences in this disease model. GSK3 $\beta$ in the active, unphosphorylated form suppresses protein synthesis by inhibiting translation initiation factor eIF2b. Phosphorylation of GSK3 $\beta$ allows translation initiation and promotes hypertrophy in cardiac myocytes (47).

We hypothesized that the soy diet increases activity of the IGF-1/ Akt/GSK3 $\beta$ cascade, which is then permissive of $\beta$-MyHC protein synthesis. Therefore, the phosphorylation state of GSK3 $\beta$ as a fraction of total GSK3 $\beta$ was measured in LV homogenates. As predicted, the HCM mice exhibited a 2- to 3-fold higher ratio of phosphorylated (inactive) to total GSK3 $\beta$ compared with WT controls (Figure 5C). However, phosphorylated GSK3 $\beta$ was over 3-fold higher in the male HCM mice fed a soy diet compared with male HCM mice fed a casein diet. This elevation correlates well with the significant increase in $\beta$-MyHC protein. While we expected to observe a higher ratio of phospho- to total GSK3 $\beta$ in the HCM mice, the diet difference in the HCM males is an unexpected and novel finding. Significantly higher phosphorylation states as observed in the HCM males consuming the soy diet may be a mechanism contributing to the transition to the decompensated state.

\section{Figure 5}

Positive adaptation is observed in male mice consuming a casein diet. $\beta$-MyHC exerts a dominant effect in heart failure and is a mechanism behind impairments in contractile function. (A) $\beta$-MyHC (expressed as a percentage of total MyHC content) is increased in hearts from 8-month-old mice consuming a soy diet. $n=3-7$ in each group. ${ }^{*} P<0.05$ versus WT same sex and diet and same sex, alternate diet HCM mice. t $P<0.05$ versus alternate sex, same diet HCM mice. (B) Representative silver-stained MyHC separation gel from WT male and HCM male mice. (C) Increased activation of the IGF-1 receptor upregulates MyHC protein in the heart. GSK3 $\beta$ is a protein kinase downstream from the IGF receptor. The ratio of phospho- to total GSK3 $\beta$ is significantly attenuated in the males consuming a phytoestrogen-free diet. The increased activity down this cascade may be permissive of increased $\beta$-MyHC protein synthesis. $n=5$ in each group. ${ }^{* *} P<0.05$ versus WT same sex and diet; ${ }^{P} P<0.05$ versus alternate diet, same sex HCM mice and alternate sex $\mathrm{HCM}$ mice regardless of diet. Mean \pm SEM.

Diet and apoptosis. One mechanism underlying cardiac dilation is myocellular apoptosis. Sex differences in apoptotic myocardial cell loss have been identified (20). Importantly, genistein induces apoptosis via caspase- 3 in a number of settings (48). For example, in human prostate cancer cell lines, genistein induces apoptosis through expression and activation of caspase-3 (49). Caspase-3 has been shown to be a proapoptotic factor in cardiac myocytes by initiating mitochondrial protein dissociation (50). An increase in programmed myocellular death therefore could underlie the development of the dilated phenotype in the male HCM mice on a soy diet. We measured caspase- 3 activity in the hearts at 8 months of age. There is a marked elevation in caspase- 3 activation in the male HCM mice consuming the soy-based diet, which is reversed in male HCM mice consuming a casein-based diet (Figure 6).

\section{Discussion}

The salient findings from the current study are as follows: (a) Diet significantly alters cardiac structure and function in WT and HCM animals; (b) The diet-dependent phenotype differs significantly between sexes at the macroscopic and cellular levels; (c) The phytoestrogens daidzein and genistein influence cardiac growth in a sex-dependent fashion; (d) Diet-dependent alterations in the

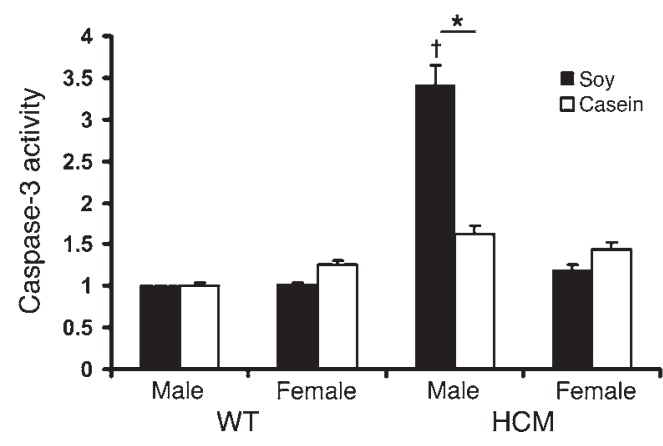

\section{Figure 6}

Increased caspase- 3 activity is indicative of increased myocellular apoptosis and may be a mechanism contributing to cardiac dilatation in male HCM mice consuming the soy diet. Caspase-3 activity is significantly attenuated in HCM males consuming the casein diet. $n=5-13$ in each group. ${ }^{*} P<0.05$ versus WT same sex and diet; t $P<0.05$ versus alternate diet, same sex HCM mice and alternate sex mice regardless of diet. Mean \pm SEM. 
IGF-1/Akt/GSK3 $\beta$ pathway, $\beta$-MyHC content, and caspase- 3 activation are potential molecular mechanisms responsible for these changes in phenotype. To our knowledge, this is the first report of significant differences in cardiac muscle adaptation due to dietary manipulation. These data strongly suggest further investigation into a link between diet and cardiomyopathy.

A major difference between the 2 diets in the current study is the protein source. The standard laboratory diet is soy based, containing significant amounts of phytoestrogens, which are major steroid receptor ligands and have been shown to have multiple biological effects. Although the relative estrogenic activity is $10^{-2}$ - to $10^{-3}$-fold less than estradiol or estrone, the principal circulating estrogens in most mammals (4), there are several characteristics that promote biological activity. These estrogenic compounds have less nonspecific binding to proteins in blood, which enhances the number of molecules available for receptor binding (51). In addition, serum concentrations of phytoestrogens with modest dietary intake have been observed to be $10^{4}$ - to $10^{5}$-fold higher than endogenous estradiol concentrations (4). Phytoestrogens also have a preference for the ER $\beta$ and several non-ER related actions (i.e., protein tyrosine kinase inhibitor and antioxidant). Perhaps most importantly, these pleiomorphic effects have been observed to occur within an individual organ or cell, consistent with steroid action in general.

We and others (52-56) have demonstrated that sex steroids influence cardiac growth. The exact mechanism of action is unclear, but additional investigation is ongoing. These results establish the biological plausibility of the influence of phytoestrogens on the heart to elicit cardiac growth via a sex steroid-receptor mechanism. While there are several reasonable theories regarding the influence of phytoestrogens on sex hormone receptors in the heart, we would propose that it is a difference in the effective estrogen dose between the sexes that may be responsible for the results of our investigation.

Since sex-dependent alterations in myocardial ERs have never been described, we would propose that the augmented estrogenic exposure in the male mice contributes to the dilated phenotype. We speculate that, because female animals have higher endogenous estrogen levels, the proportional increase in estrogenic compounds via diet is less in females compared with males, who are chronically exposed to significantly lower levels of estrogenic compounds. For example, it has been estimated that basal female $17 \beta$-estradiol levels are $5-30 \mathrm{pg} / \mathrm{ml}$ while males have negligible levels (57-60). Serum phytoestrogen levels have been reported in the $1000-2000 \mathrm{ng} / \mathrm{ml}$ range on several standard rodent diets independent of $\operatorname{sex}(61)$.

Differences in affinity and estrogenic activity between phytoestrogens and endogenous estrogens make it difficult to assess the proportion of biological activity due to each individual compound in female animals. In males this is not the case since endogenous estrogenic compounds are present only in negligible quantities; therefore the full effect of the phytoestrogens would be observed. To support this argument further, Boettger-Tong et al. have demonstrated a failure of the usual biochemical and morphological uterine responses to exogenously administered estradiol in animals that were fed a rodent diet with soy meal and alfalfa (62). This occurred when the rodent diet had been reformulated without any investigator notification. Since, as noted above, isoflavone levels have not been demonstrated to be different between males and females on identical diets, the absolute influence of these compounds should be substantially greater in the male animals that have virtually no basal estrogenic stimulation. Moreover, there is in vivo evidence that genistein acts as an agonist at ERs when administered alone and as an antagonist when coadministered with $17 \beta$-estradiol (63). It is plausible that a similar mechanism is behind the sex difference in cardiac phenotype.

Indeed, our results demonstrate that phytoestrogen supplementation augments cardiac growth in the HCM males and attenuates growth in the HCM females. The current results do not make any assumptions regarding the molecular and functional phenotype of the HCM mice on the supplemented diet. Importantly, adding phytoestrogens to the casein diet does not recapitulate the entire cardiac phenotype of the young animals consuming the soy diet. This is not surprising given that the dietary milieu is complex and unregulated in the soy diet. There are clearly other components in the diet that influence cardiac growth. This fact accentuates the importance of dietary intake in other experimental models. However, it is beyond the scope of the current study to differentiate the other components of the soy diet that may contribute to the sex difference in phenotype.

Phytoestrogens also activate PPAR $\alpha$, which is downregulated in heart failure in vivo. In our genetic model, we observed increased cardiac growth in animals consuming a soy-free diet, suggesting that PPAR $\alpha$ is not contributing to the improvement in the phenotype in our model. However, the casein diet was also associated with an improvement in cardiac function assessed by echocardiography in the absence of a change in systemic blood pressure. Importantly, the increased growth was accompanied by improved cellular markers of disease including lower $\beta$-MyHC expression and improved cellular architecture (less thick collagen deposition and less myocellular disarray).

The soy-based laboratory diet was associated with increased caspase- 3 activity and increased activity through the IGF-1/Akt/ GSK3 $\beta$ pathway in the diseased animals relative to WT. IGF-1 stimulation is associated with increased $\beta$-MyHC synthesis in rat models (64). Augmentation of this pathway may lead directly to the systolic impairment observed in the male HCM mice on the soy diet. Alternatively, the increased activity along this pathway may be a secondary phenomenon in response to the differences in cardiac growth. Future directions will include defining the role of this pathway by crossbreeding the HCM model with a constitutively active GSK transgenic mouse (44) and identification of a common upstream activator. The increase in caspase-3 activity in HCM hearts on a soy diet indicates elevated levels of myocellular death, or apoptosis. Preliminary data from our laboratory (data not shown) demonstrate that the increase in caspase- 3 activity was associated with decreased levels of Bcl-2 (an inhibitor of apoptosis) and pro-caspase-9 (a precursor of effector caspases). These additional preliminary results further support the conclusion that there is augmented apoptosis in the HCM mice on a soy diet. Genistein has been shown to induce apoptosis via activated caspase-3 in a number of settings $(48,49)$. An increase in apoptosis is likely an important contributor to the adverse remodeling observed in dilated cardiomyopathy in human clinical populations and may be responsible in part for the transition from the compensated hypertrophic to the decompensated dilated state in the mouse model.

Our results suggest that the dilated phenotype observed in the male HCM mice on the soy diet is a result of an augmented growth pathway and an augmented programmed cell death pathway. It is likely that the decompensated phenotype results from a transition from a balance to an imbalance of cell growth and cell death. 
Components of the soy diet have been associated with both myocellular hypertrophy and apoptosis in several other model systems and may be modifiers of the balance between these 2 processes in vivo. Investigation of functional and molecular changes occurring in the hearts of mice on the phytoestrogen-supplemented diet will further elucidate the influence of phytoestrogens on cardiac disease. Additional investigation into other specific components of the soy diet as well as identification of characteristics of these pathways during the compensated state are necessary to fully elucidate these mechanistically.

Taken together these data show an increase in nonpathologic, or physiologic, cardiac growth in this HCM mouse model on a soy-free diet. The modifiers of caspase activity and the IGF-1/Akt/ GSK3 $\beta$ hypertrophic pathway are unknown but deserve additional attention. This discovery may lead to novel dietary modifications that prevent progression or formation of some hypertrophic cardiac diseases. Future studies will be directed against identifying specific genetic modifiers that affect the response of the heart to diet. These will include testing the effect of diet on different strains of inbred mice. There is a large literature of human studies in which correlations have been made between certain diets and diseases, particularly cancer (3). And, in some cases, the results have been inconclusive. The kind of study reported here on genetically identical groups of mice of both sexes should address the issue of genetic variability in the human studies and begin to define the role that genes play in dietary responses.

\section{Methods}

Animals. The HCM mouse model used in this study expressed a mutant rat $\alpha-\mathrm{MyHC}$ with expression driven by an $\alpha-\mathrm{MyHC}$ promoter (29). The transgene coding region contained 2 mutations, a point mutation, R403Q, and a deletion of 59 amino acids in the actin binding site bridged by the addition of 9 nonmyosin amino acids. Male and female WT and HCM mice were fed a soy diet, a casein (phytoestrogen-free) diet, or a casein diet supplemented with phytoestrogens ad libitum. Mice were genotyped by PCR from a 2 -mm tail sample prior to study inclusion. Given the large number of animals included in this study, repeat genotyping was performed on a separate tissue sample at the time of animal sacrifice to assure correct grouping. There were no misidentified animals noted. All animal protocols were approved by the Institutional Animal Care and Use Committee at the University of Colorado at Boulder and at the University of Colorado Health Sciences Center.

Diets. The formulation of the casein diet (D10001; Research Diets) follows the guidelines recommended by the American Institute of Nutrition in 1977 (65). The soy diet used in the present study was the Harlan Teklad Sterilizable Rodent Diet (8656; Harlan Teklad). The casein and soy diets are composed of approximately the same percentage of protein (20-24\%), fat $(4-5 \%)$, carbohydrate $(65 \%)$, and fiber $(5 \%)$ with similar amounts of vitamins and minerals. Differences between the 2 diets, however, include the source of protein (casein in the D10001 versus alfalfa and soybean in the 8656 diet), carbohydrate (sucrose and corn starch in the D10001 versus corn and soybean meal in the 8656 diet), and fat (corn oil in the D10001 versus soybean oil in the 8656 diet). The effective caloric content was different between the diets as well (D10001, $3.9 \mathrm{kcal} / \mathrm{g}$ versus 8656, $3.25 \mathrm{kcal} / \mathrm{g}$ ). However, total caloric consumption was similar between groups since the animals consuming the soy diet consumed more food (approximately $10 \%$ more). The isoflavone concentration of the soy-based rodent diet was determined at $206 \mathrm{mg}$ isoflavone/ $\mathrm{kg}$ dry food weight daidzein and 229.5 $\mathrm{mg}$ isoflavone/kg dry food weight genistein. The phytoestrogen supplementation diet was created by adding comparable amounts of daidzein and genistein (LC Laboratories) to the casein diet.
Surgical gonadectomy. At 1 month of age, mice were anesthetized with $2.5 \%$ tribromoethanol (Avertin) by intraperitoneal injection. Females underwent bilateral oophorectory through bilateral paraspinal incisions. Males underwent orchiectomy via a transverse scrotal incision. The incisions were repaired with suture and skin staples. While still under anesthesia, sustained release (90 day) hormone pellets (Innovative Research of America) of $17 \beta$-estradiol, testosterone, or vehicle placebo were implanted subcutaneously in the nape of the neck. Sham-operated animals were handled identically except the gonads were not removed.

Gravimetric measurements. At 2 or 8 months of age, mice were euthanized using approved methods. Each mouse was weighed, and the length of the right tibia was recorded. Hearts were rapidly excised and washed with icecold normal saline $(9 \% \mathrm{NaCl} \mathrm{w} / \mathrm{v})$. The great vessels and all atrial tissue were removed under a dissecting scope (Carl Zeiss), and the ventricles were blotted dry and weighed. The RVs and LVs were rapidly separated and individually flash frozen in liquid nitrogen. The samples were stored at $-80^{\circ} \mathrm{C}$ until further analysis was performed.

Echocardiography. Echocardiography was performed on animals at 8 months of age as previously described (66). A VingMed System Five echocardiography machine (GE Medical Systems) with a 10-MHz-phased array transducer was used for digital image acquisition. The mice were positioned prone, and M-mode recordings were obtained and saved on magnetic optical media for offline analysis, which was performed using EchoPAC software (version 6; GE Medical Systems). Fractional shortening was calculated using the average over 3 cardiac cycles.

Blood pressure measurement. In vivo systemic blood pressure measurement was performed on animals at 8 months of age. Each mouse was injected intraperitoneally immediately before evaluation with $2.5 \%$ tribromoethanol (Avertin). A longitudinal skin incision was performed over the trachea, and the right carotid artery was isolated using blunt dissection under $\times 6.5$ magnification (Carl Zeiss). The cephalad portion of the carotid artery was ligated at the base of the skull. A small nick was made in the midportion of the artery, and a 1.4-fr Millar Mikro-tip pressure transducer (Millar Instruments) was advanced through the nick and into the aorta. After a period of equilibration, aortic pressures were recorded. Following blood pressure measurements, the animals were euthanized.

Histology. Following euthanasia at 8 months of age, hearts were rapidly excised and washed, and whole-heart weight was recorded. The entire heart was placed in $10 \%$ neutral-buffered formalin for 24 hours for fixation prior to histological evaluation. The fixed hearts were processed, embedded in paraffin, sectioned, and stained with picrosirius red according to standard protocols. Thin collagen fibers showed green birefringence, and thick collagen fibers showed bright yellow/orange birefringence under polarization light microscopy. Myofibrils in series exhibited slight birefringence.

Western blot analysis. Frozen LV tissue was homogenized on ice in a protein extraction buffer: $\mathrm{NaCl}(137 \mathrm{mM})$; Tris(hydroxymethyl)-aminomethane (20 mM); 10\% vol/vol glycerol; $1 \%$ vol/vol NP-40, pH 7.4. The homogenized tissue was centrifuged at $12,000 \mathrm{~g}$ for 10 minutes at $4^{\circ} \mathrm{C}$ and the supernatant removed. Protein concentration was determined using the Bradford method. SDS-PAGE separation was performed under denaturing conditions. The proteins were transferred to a PVDF membrane (Amersham Biosciences) using standard techniques. The membranes were probed with antibodies (Santa Cruz Biotechnology) specific for either total GSK3 $\beta$ or the phosphorylated isoform. Immunoreactivity was visualized using a Western Lightning chemiluminescence detection system (PerkinElmer) and quantified using densitometry.

MyHC expression. LV tissue was homogenized in a myosin sample buffer: urea $(8 \mathrm{~mol} / \mathrm{l})$; thiourea $(2 \mathrm{~mol} / \mathrm{l})$; Tris, $\mathrm{pH} 6.8$ (0.05 mol/l); dithiothreitol (DTT) $(0.075 \mathrm{~mol} / \mathrm{l}) ; 3 \% \mathrm{SDS}$. Each sample was heated at $100^{\circ} \mathrm{C}$ for 3 minutes and then loaded in 6 wells containing escalating amounts of total 
protein ( 0.5 to $50 \mu \mathrm{g} /$ well). The samples were separated by $6 \%$ SDS-PAGE at $16 \mathrm{~mA}$ for 4.5 hours at $8^{\circ} \mathrm{C}$. Gels were silver stained, and the percentages of $\alpha$ - and $\beta$-MyHC protein were determined by densitometry (67).

Caspase- 3 activity assay. Caspase- 3 activity was determined by monitoring the rate of cleavage of a fluorogenic caspase-3 specific substrate (Acetyl-AspGluValAsp-AMC; Calbiochem). To do this, frozen hearts were mechanically disrupted in an ice-cold lysis buffer $(0.02 \mathrm{ml} / \mathrm{g}$ tissue): Tris(hydroxymethyl)-aminomethane (20 mM); $\mathrm{NaCl}(137 \mathrm{mM})$; EDTA (0.2 mM); EGTA (0.5 mM); Triton X-100 (1\%); glycerol (10\%), pH 7.4. In a 96-well plate, $0.5 \mathrm{mg}$ of protein was added to each well with an equal volume $(50 \mu \mathrm{l})$ of caspase- 3 activity assay buffer containing: Tris(hydroxymethyl)-aminomethane (50 mM); EDTA ( $0.5 \mathrm{mM})$; glycerol (20\%); caspase- 3 substrate $(0.02 \mathrm{mM})$; DTT $(0.004 \mathrm{mM})$, pH 7.0. Cleavage of the substrate was monitored by excitation at $380 \mathrm{~nm}$ and emission at $460 \mathrm{~nm}$ with a Fluorskan Ascent Microplate Fluorometer (Thermo Electron Corp.). Caspase-3 activity was determined by calculating the slope of the linear portion of the cleaved substrate and then normalized to protein content (fluorescent units $/ \mathrm{min} / \mathrm{mg}$ protein).

Data and statistical analysis. Results are presented as mean \pm SEM. Between group differences of morphometric, protein, and blood pressure, data were determined using a 2 between (male, female) by 2 between (soy, casein) by 2 between (WT, HCM) ANOVA at 8 months of age. Between group dif- ferences of echocardiographic indices were determined using a 2 between (male, female) by 2 between (soy, casein) ANOVA. Dietary between group differences were determined using a 2 between (male, female) by 3 between (soy, casein, supplemented) ANOVA. Interactions and/or specific main effects differences were examined by Tukey post hoc analyses. $P<0.05$ was considered significant a priori.

\section{Acknowledgments}

This work was supported by grants from the NIH (2R01HL050560 to L.A. Leinwand; 5F32HL070509 to J.P. Konhilas; and 1F32HL67543 to B.L. Stauffer) and the American Heart Association (0120679Z to B.L. Stauffer). Isoflavone concentrations of the soy-based rodent diet were kindly determined by Patricia A. Murphy, Iowa State University, Ames, Iowa.

Received for publication February 8, 2005, and accepted in revised form October 18, 2005.

Address correspondence to: Leslie A. Leinwand, Molecular, Cellular, and Developmental Biology, Room A417, UCB 354, Boulder, Colorado 80309, USA. Phone: (303) 492-7606; Fax: (303) 4928907; E-mail: Leslie.Leinwand@colorado.edu.
1. Ordovas, J.M., and Corella, D. 2004. Nutritional genomics. Annu. Rev. Genomics Hum. Genet. 5:71-118.

2. Grierson, B. 2003. What your genes want you to eat. The New York Times Magazine. May 4. 76-77.

3. Glazier, M.G., and Bowman, M.A. 2001. A review of the evidence for the use of phytoestrogens as a replacement for traditional estrogen replacement therapy. Arch. Intern. Med. 161:1161-1172.

4. Setchell, K.D. 1998. Phytoestrogens: the biochemistry, physiology, and implications for human health of soy isoflavones. Am. J. Clin. Nutr. 68:1333S-1346S.

5. Squadrito, F., et al. 2003. Effect of genistein on endothelial function in postmenopausal women: a randomized, double-blind, controlled study. Am. J. Med. 114:470-476.

6. Sacks, F.M. 2005. Dietary phytoestrogens to prevent cardiovascular disease: early promise unfulfilled. Circulation. 111:385-387.

7. Cornwell, T., Cohick, W., and Raskin, I. 2004. Dietary phytoestrogens and health. Phytochemistry. 65:995-1016.

8. Doerge, D.R., and Chang, H.C. 2002. Inactivation of thyroid peroxidase by soy isoflavones, in vitro and in vivo. J. Chromatogr. B Analyt. Technol. Biomed. Life Sci. 777:269-279.

9. McVey, M.J., Cooke, G.M., and Curran, I.H. 2004. Increased serum and testicular androgen levels in F1 rats with lifetime exposure to soy isoflavones. Reprod. Toxicol. 18:677-685.

10. Barnes, S. 2004. Soy isoflavones-phytoestrogens and what else? J. Nutr. 134:1225S-1228S.

11. Mezei, O., et al. 2003. Soy isoflavones exert antidiabetic and hypolipidemic effects through the PPAR pathways in obese Zucker rats and murine RAW 264.7 cells. J. Nutr. 133:1238-1243.

12. Kim, S., et al. 2004. Genistein enhances expression of genes involved in fatty acid catabolism through activation of PPARalpha. Mol. Cell. Endocrinol. 220:51-58.

13. Sack, M.N., et al. 1996. Fatty acid oxidation enzyme gene expression is downregulated in the failing heart. Circulation. 94:2837-2842.

14. Karbowska, J., Kochan, Z., and Smolenski, R.T. 2003. Peroxisome proliferator-activated receptor alpha is downregulated in the failing human heart. Cell. Mol. Biol. Lett. 8:49-53.

15. van der Schouw, Y.T., et al. 2005. Prospective study on usual dietary phytoestrogen intake and cardio- vascular disease risk in western women. Circulation. 111:465-471.

16. Aronow, W.S., Ahn, C., and Kronzon, I. 1999. Comparison of incidences of congestive heart failure in older African-Americans, Hispanics, and whites. Am. J. Cardiol. 84:611-612, A619.

17. Aurigemma, G.P., and Gaasch, W.H. 1995. Gender differences in older patients with pressure-overload hypertrophy of the left ventricle. Cardiology. 86:310-317.

18. Krumholz, H.M., Larson, M., and Levy, D. 1993. Sex differences in cardiac adaptation to isolated systolic hypertension. Am. J. Cardiol. 72:310-313.

19. Greaves, S.C., Roche, A.H., Neutze, J.M., Whitlock, R.M., and Veale, A.M. 1987. Inheritance of hypertrophic cardiomyopathy: a cross sectional and $\mathrm{M}$ mode echocardiographic study of 50 families. $\mathrm{Br}$. Heart J. 58:259-266.

20. Olivetti, G., et al. 1995. Gender differences and aging: effects on the human heart. J. Am. Coll. Cardiol. 26:1068-1079.

21. Pelzer, T., Shamim, A., Wolfges, S., Schumann, M., and Neyses, L. 1997. Modulation of cardiac hypertrophy by estrogens. In Hypertension and the heart. Zanchetti, A., editor. Plenum Press. New York, New York, USA. 83-89.

22. Ho, K.K., Anderson, K.M., Kannel, W.B., Grossman, W., and Levy, D. 1993. Survival after the onset of congestive heart failure in Framingham Heart Study subjects. Circulation. 88:107-115.

23. Levy, D., et al. 2002. Long-term trends in the incidence of and survival with heart failure. N. Engl. J. Med. 347:1397-1402.

24. Anderson, G.L., et al. 2004. Effects of conjugated equine estrogen in postmenopausal women with hysterectomy: the Women's Health Initiative randomized controlled trial. JAMA. 291:1701-1712.

25. Rossouw, J.E., et al. 2002. Risks and benefits of estrogen plus progestin in healthy postmenopausal women: principal results from the Women's Health Initiative randomized controlled trial. JAMA. 288:321-333.

26. Marsh, J.D., et al. 1998. Androgen receptors mediate hypertrophy in cardiac myocytes. Circulation. 98:256-261.

27. Grohe, C., et al. 1997. Cardiac myocytes and fibroblasts contain functional estrogen receptors. FEBS Lett. 416:107-112.
28. Olsson, M.C., Palmer, B.M., Leinwand, L.A., and Moore, R.L. 2001. Gender and aging in a transgenic mouse model of hypertrophic cardiomyopathy. Am. J. Physiol. Heart Circ. Physiol. 280:H1136-H1144.

29. Vikstrom, K.L., Factor, S.M., and Leinwand, L.A. 1996. Mice expressing mutant myosin heavy chains are a model for familial hypertrophic cardiomyopathy. Mol. Med. 2:556-567.

30. Thigpen, J.E., et al. 1999. Phytoestrogen content of purified, open- and closed-formula laboratory animal diets. Lab. Anim. Sci. 49:530-536.

31. Thigpen, J.E., Setchell, K.D., Goelz, M.F., and Forsythe, D.B. 1999. The phytoestrogen content of rodent diets. Environ. Health Perspect. 107:A182-A183.

32. Johnson, B.D., et al. 1997. Increased expression of the cardiac L-type calcium channel in estrogen receptordeficient mice. J. Gen. Physiol. 110:135-140.

33. Xu, Y., Arenas, I.A., Armstrong, S.J., and Davidge, S.T. 2003. Estrogen modulation of left ventricular remodeling in the aged heart. Cardiovasc. Res. 57:388-394.

34. Jalil, J.E., et al. 1989. Fibrillar collagen and myocardial stiffness in the intact hypertrophied rat left ventricle. Circ. Res. 64:1041-1050.

35. Hess, O.M., et al. 1981. Diastolic function and myocardial structure in patients with myocardial hypertrophy. Special reference to normalized viscoelastic data. Circulation. 63:360-371.

36. Kawai, S., Okada, R., Kitamura, K., Suzuki, A., and Saito, S. 1984. A morphometrical study of myocardial disarray associated with right ventricular outflow tract obstruction. Jpn. Circ. J. 48:445-456.

37. Swynghedauw, B. 1986. Developmental and functional adaptation of contractile proteins in cardiac and skeletal muscles. Physiol. Rev. 66:710-771.

38. Tardiff, J.C., et al. 2000. Expression of the beta (slow)-isoform of MHC in the adult mouse heart causes dominant-negative functional effects. Am.J. Physiol. Heart Circ. Physiol. 278:H412-H419.

39. Herron, T.J., Korte, F.S., and McDonald, K.S. 2001. Loaded shortening and power output in cardiac myocytes are dependent on myosin heavy chain isoform expression. Am. J. Physiol. Heart Circ. Physiol. 281:H1217-H1222.

40. Florini, J.R., Ewton, D.Z., and Foster, J.A. 1991. Modern concepts of insulin-like growth factors. Elsevier. New York, New York, USA. 487-503.

41. Decker, R.S., Cook, M.G., Behnke-Barclay, M., and 
Decker, M.L. 1995. Some growth factors stimulate cultured adult rabbit ventricular myocyte hypertrophy in the absence of mechanical loading. Circ. Res. 77:544-555.

42. Camper-Kirby, D., et al. 2001. Myocardial Akt activation and gender: increased nuclear activity in females versus males. Circ. Res. 88:1020-1027.

43. Matsui, T., et al. 2002. Phenotypic spectrum caused by transgenic overexpression of activated Akt in the heart. J. Biol. Chem. 277:22896-22901.

44. Antos, C.L., et al. 2002. Activated glycogen synthase- 3 beta suppresses cardiac hypertrophy in vivo. Proc. Natl. Acad. Sci. U. S. A. 99:907-912.

45. Klotz, D.M., et al. 2002. Requirement of estrogen receptor-alpha in insulin-like growth factor-1 (IGF-1)induced uterine responses and in vivo evidence for IGF-1/estrogen receptor cross-talk. J. Biol. Chem. 277:8531-8537.

46. Martin, M.B., et al. 2000. A role for Akt in mediating the estrogenic functions of epidermal growth factor and insulin-like growth factor I. Endocrinology. 141:4503-4511.

47. Haq, S., et al. 2000. Glycogen synthase kinase-3beta is a negative regulator of cardiomyocyte hypertrophy. J. Cell Biol. 151:117-130.

48. Choi, E.J., and Lee, B.H. 2004. Evidence for genistein mediated cytotoxicity and apoptosis in rat brain. Life Sci. 75:499-509.

49. Kumi-Diaka, J., Sanderson, N.A., and Hall, A. 2000. The mediating role of caspase- 3 protease in the intracellular mechanism of genistein-induced apoptosis in human prostatic carcinoma cell lines, DU145 and LNCaP. Biol. Cell. 92:595-604.

50. Thornberry, N.A., and Lazebnik, Y. 1998. Caspases: enemies within. Science. 281:1312-1316.

51. Nagel, S.C., vom Saal, F.S., and Welshons, W.V. 1998 The effective free fraction of estradiol and xenoestrogens in human serum measured by whole cell uptake assays: physiology of delivery modifies estrogenic activity. Proc. Soc. Exp. Biol. Med. 217:300-309.

52. Sharkey, L.C., et al. 1998. Effect of ovariectomy in heart failure-prone SHHF/Mcc-facp rats. Am. J. Physiol. 275:R1968-R1976.

53. Sharkey, L.C., et al. 1999. Effect of ovariectomy and estrogen replacement on cardiovascular disease in heart failure-prone SHHF/Mcc- fa cp rats. J. Mol. Cell. Cardiol. 31:1527-1537.

54. Schaible, T.F., Malhotra, A., Ciambrone, G., and Scheuer, J. 1984. The effects of gonadectomy on left ventricular function and cardiac contractile proteins in male and female rats. Circ. Res. 54:38-49.

55. Gao, X.M., et al. 2003. Sex hormones and cardiomyopathic phenotype induced by cardiac beta 2 adrenergic receptor overexpression. Endocrinology. 144:4097-4105.

56. Malhotra, A., Buttrick, P., and Scheuer, J. 1990. Effects of sex hormones on development of physiological and pathological cardiac hypertrophy in male and female rats. Am. J. Physiol. 259:H866-H871.

57. Barkley, M.S., Lasley, B.L., Thompson, M.A., and Shackleton, C.H. 1985. Equol: a contributor to enigmatic immunoassay measurements of estrogen. Steroids. 46:587-608.

58. Butcher, R.L., Collins, W.E., and Fugo, N.W. 1974. Plasma concentration of $\mathrm{LH}, \mathrm{FSH}$, prolactin, progesterone and estradiol-17beta throughout the 4-day estrous cycle of the rat. Endocrinology. 94:1704-1708.
59. Nequin, L.G., Alvarez, J., and Schwartz, N.B. 1979. Measurement of serum steroid and gonadotropin levels and uterine and ovarian variables throughout 4 day and 5 day estrous cycles in the rat. Biol. Reprod. 20:659-670.

60. Loeb, W.F., and Quimby, F.W. 1999. The clinical chemistry of laboratory animals. 2nd edition. Taylor \& Francis Group. Philadelphia, Pennsylvania, USA. 753 pp.

61. Brown, N.M., and Setchell, K.D. 2001. Animal models impacted by phytoestrogens in commercial chow: implications for pathways influenced by hormones. Lab. Invest. 81:735-747.

62. Boettger-Tong, H., et al. 1998. A case of a laboratory animal feed with high estrogenic activity and its impact on in vivo responses to exogenously administered estrogens. Environ. Health Perspect. 106:369-373

63. Ratna, W.N. 2002. Inhibition of estrogenic stimulation of gene expression by genistein. Life Sci. 71:865-877.

64. Morkin, E. 2000. Control of cardiac myosin heavy chain gene expression. Microsc. Res. Tech. 50:522-531.

65. [Anonymous]. 1977. Report of the American Institute of Nutrition ad hoc Committee on Standards for Nutritional Studies. J. Nutr. 107:1340-1348.

66. Freeman, K., et al. 2001. Progression from hypertrophic to dilated cardiomyopathy in mice that express a mutant myosin transgene. Am. J. Physiol. Heart Circ. Physiol. 280:H151-H159.

67. Olsson, M.C., Palmer, B.M., Stauffer, B.L., Leinwand, L.A., and Moore, R.L. 2004. Morphological and functional alterations in ventricular myocytes from male transgenic mice with hypertrophic cardiomyopathy. Circ. Res. 94:201-207. 